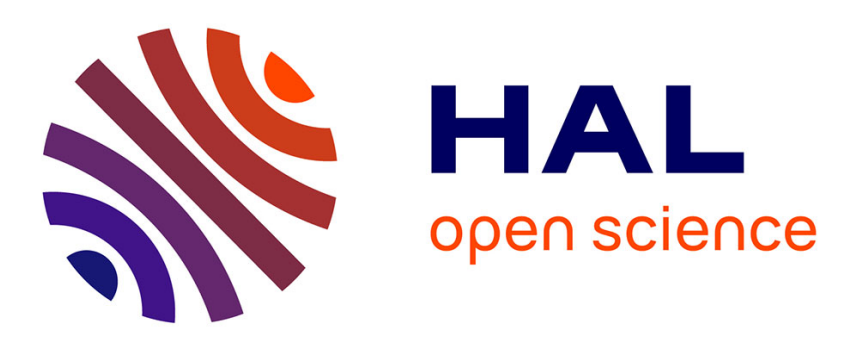

\title{
Temperature-driven coherence resonance and stochastic resonance in a thermochemical system
}

\author{
Annie Lemarchand, J Gorecki, A Gorecki, B Nowakowski
}

\section{To cite this version:}

Annie Lemarchand, J Gorecki, A Gorecki, B Nowakowski. Temperature-driven coherence resonance and stochastic resonance in a thermochemical system. Physical Review E : Statistical, Nonlinear, and Soft Matter Physics, 2014, 89 (2-1), pp.022916. 10.1103/PhysRevE.89.022916 . hal-01089935

\section{HAL Id: hal-01089935 \\ https://hal.sorbonne-universite.fr/hal-01089935}

Submitted on 2 Dec 2014

HAL is a multi-disciplinary open access archive for the deposit and dissemination of scientific research documents, whether they are published or not. The documents may come from teaching and research institutions in France or abroad, or from public or private research centers.
L'archive ouverte pluridisciplinaire HAL, est destinée au dépôt et à la diffusion de documents scientifiques de niveau recherche, publiés ou non, émanant des établissements d'enseignement et de recherche français ou étrangers, des laboratoires publics ou privés. 


\title{
Temperature driven coherence resonance and stochastic resonance in a thermochemical system
}

\author{
A. Lemarchand ${ }^{a, b}$, J. Goreckic,d ${ }^{*}$ A. Gorecki ${ }^{e}$, and B. Nowakowski ${ }^{c, e}$ \\ ${ }^{a}$ Université Pierre et Marie Curie - Paris 6,
}

Laboratoire de Physique Théorique de la Matière Condensée (LPTMC), 4 place Jussieu, case courrier 121, 75252 Paris Cedex 05, France

${ }^{b}$ CNRS, LPTMC, UMR 7600, Paris, France

${ }^{c}$ Institute of Physical Chemistry, Polish Academy of Sciences,

Kasprzaka 44/52, 01-224 Warsaw, Poland

${ }^{d}$ Faculty of Mathematics and Life Science, UKSW, Warsaw, Poland

e Physics Laboratory, Warsaw University of Life Sciences,

Nowoursynowska 159, 02-776, Warsaw, Poland

* E-mail: jgorecki@ichf.edu.pl

January 29, 2014 


\begin{abstract}
We perform the stochastic analysis of a thermochemical system using a master equation which describes a chemical reaction and includes discrete and continuous temperature jumps. We study the time evolution of the system selecting the temperature of the thermostat as an easily tunable control parameter. Depending on the thermostat temperature, the system can be in an excitable, oscillatory or stationary regime. Stochastic time series for the system temperature are generated and the distributions of interspike intervals are analyzed in the three dynamical regimes separated by a homoclinic bifurcation and a Hopf bifurcation. Different constructive roles of internal fluctuations are exhibited. A noise-induced transition is observed in the vicinity of the Hopf bifurcation. Coherence resonance and stochastic resonance are found in the oscillatory regime. In a range of thermostat temperatures, a non trivial behavior of the highly nonlinear system is revealed by the existence of both a minimum and a maximum in the scaled standard deviation of interspike intervals as a function of particle number. This high sensitivity to system size illustrates that controlling dynamics in nanoreactors may remain a difficult task.
\end{abstract}

PACS: 82.40.Bj, 05.10.Gg, 82.33.Vx 


\section{Introduction}

The elucidation of biochemical mechanisms at the cellular level and the design of nanoreactors represent strong motivations to develop submicrometric descriptions of reactive, far-from-equilibrium systems. In small chemical systems or in systems containing a small number of molecules, the internal fluctuations can induce deviations from the deterministic predictions that are especially important in specific conditions, such as the vicinity of bifurcations [1] or when the propagation of a chemical wave front is involved [2]. More generally, the constructive effects of noise have been pointed out in diverse situations, such as stochastic resonance $[3,4,5,6,7]$, coherence resonance $[8,9,10,11]$, Brownian motors [12], and noise-induced order in chaotic systems [13]. Chemical processes are rarely thermoneutral and, at small scales, the description of temperature variation can be hardly ignored. For example, in the case of even slightly exothermal chemical wave fronts, noise-sensitive abrupt transitions to detonation may be observed, leading to unexpected very fast propagation of the front [14].

The description of fluctuations in isothermal reaction-diffusion systems is well known. Reaction and diffusion are considered as Markovian processes associated with discrete jumps for the number of particles and master equations that describe these processes can be easily written $[15,16,17]$ and solved [18]. In contrast, stochastic approaches including temperature as a random variable are not simple because temperature undergoes continuous, unbounded jumps when the system exchanges energy with the surrounding. Different stochastic descriptions of thermochemical systems have been pro- 
posed $[19,20,21]$. We have developed an original approach based on the kinetic theory [22] and defined the transition probabilities associated with exothermal reactive processes and exchanges with a thermostat [23]. Kinetic Monte Carlo simulations of the resulting integro-differential master equation associated with various typical thermochemical systems have revealed non trivial effects of temperature fluctuations.

Noise-induced transitions $[1,6]$ modifying the bifurcation diagram deduced from the deterministic description have been reported for bistable thermochemical systems. In such cases, predictions of the master equation have been successfully compared to microscopic simulations based on the direct simulation Monte Carlo method [24, 25]. If a system is close to a Hopf bifurcation, then, for an appropriate amplitude of the internal fluctuations controlled by system size, a coherence resonance [26] leading to the better synchronisation of fluctuations around the steady state has been characterized [27].

We already reported on the existence of coherence resonance in a thermochemical system with one or three stationary states [27, 28, 29, 30]. The macroscopic description of the system introduces different control parameters: rate constants, the reaction heat and the thermostat temperature. In the stochastic description, the amplitude of the internal fluctuations also depends on the total number of particles. Up to now, we proved the existence of a minimum for the standard deviation of the interspike interval scaled by the mean interval as the number of particles or reaction heat varies [27, 28, 29]. We have recently shown [30] that, for both the number of molecules and the 
reaction heat regarded as control parameters, coherence resonance is accompanied by information resonance, i. e. by a minimum of Shannon entropy [31]. The influence of product conversion rate on the stochastic evolution of the thermochemical model has also been studied [27]. Nevertheless, it seems difficult to design an experiment in which a reaction heat or a reaction rate can be used as a control parameter. Such an experiment should be based on different chemical reactions, which would imply different systems, but not simply the variation of a parameter for a single one. On the contrary, the thermostat temperature, that can be fixed at will for a given reactive system, is the best candidate for an experimental validation of fluctuation effects on exothermal reactions. Although thermostat temperature appears as a natural control parameter, theoretical studies of its influence on stochastic effects in thermochemical systems have not been reported yet. Furthermore, experimental observations of coherence resonances did not involve temperature as a dynamical variable $[32,33]$.

In this paper, we investigate the behavior of a thermochemical system as thermostat temperature or system size varies and focus on stochastic effects such as noise-induced transitions, coherence resonances and stochastic resonances. The vicinity of a homoclinic bifurcation and the vicinity of a Hopf bifurcation are studied. The possibility to detect order in the stochastic time evolution using information theory and Shannon entropy is discussed [31].

The paper is organized as follows. In Section 2, we recall the thermochemical model. For the selected values of reaction parameters, the deterministic analysis indicates the presence of a homoclinic bifurcation and a Hopf bi- 
furcation as thermostat temperature varies. The master equation associated with the chemical and thermal processes is given. The results are discussed in Section 3 for the three domains of thermostat temperature associated with excitable, oscillating and stationary regimes. The behavior as size varies is analyzed. Conclusions are presented in Section 4.

\section{The thermochemical model}

We consider a thermochemical model [34], inspired from a model introduced by Volter and Salnikov [35], which describes a perfectly mixed gaseous system composed of a reactant $\mathrm{A}$, a product $\mathrm{B}$, and a catalyst $\mathrm{C}$, engaged in the following reactions:

$$
\begin{array}{rll}
\mathrm{A}+\mathrm{C} & \stackrel{k_{1}}{\rightarrow} & \mathrm{B}+\mathrm{C}+\text { heat } q \\
\mathrm{~B} & \stackrel{k_{2}}{\rightarrow} & \mathrm{A}
\end{array}
$$

The exothermic reaction (1) proceeds in bulk and is accompanied by a heat release $q$. The second reaction occurs on the surface and mimics unspecified mechanisms of reactant supply and product removal. The energy balance of the two reactions can be positive, since the system is open to energy exchange through its boundary with a surrounding thermostat at fixed temperature $T_{b}$. At the macroscopic level, a state of the system is characterized by two variables, the number density $n_{A}$ of species $\mathrm{A}$ and temperature, $T$. The number density, $n_{C}$, of the catalyst $\mathrm{C}$ does not change in time and the total number density, $n$, also remains constant. Deterministic dynamics is governed by the following balance equations for a system of $N$ particles confined by volume 
$V$ and surface $S$ :

$$
\begin{aligned}
V \frac{d n_{A}}{d t^{\prime}} & =-k_{1} n_{A} n_{C} V+k_{2} n_{B} S \\
\frac{3}{2} n V k_{B} \frac{d T}{d t^{\prime}} & =k_{1} n_{A} n_{C} V q-\kappa n S k_{B}\left(T-T_{b}\right)
\end{aligned}
$$

where $\kappa$ is the coefficient of heat exchange of Newton's law of cooling and $k_{B}$ denotes the Boltzmann constant. Equation (3) describes the time evolution of the number density of $A$ and Eq. (4) represents the time evolution of the total energy, equal to $3 / 2 N k_{B} T$ as for an ideal gas containing $N$ particles.

Equations $(3,4)$ describe an activator-inhibitor system in which temperature plays the role of activator and number density, of inhibitor $[34,36]$. We assume that reaction (1) is thermally activated. Its rate constant $k_{1}$ is described by Arrhenius law and involves an activation energy $E_{A}$. Reaction (2) is a non-activated process associated with the rate constant $k_{2}$. The kinetic theory of gases $[22,23]$ leads to the following dependence of the rate constants and coefficient of heat exchange on temperature:

$$
\begin{aligned}
k_{1} & =k_{1}^{0} \sqrt{T} \exp \left(\frac{-E_{A}}{k_{B} T}\right) \\
k_{2} & =p_{2} \kappa \\
\kappa & =\kappa^{0} \sqrt{T}
\end{aligned}
$$

where the constants $k_{1}^{0}$ and $\kappa^{0}$ are independent of temperature and $p_{2}$ is the coefficient determining the probability of reaction (2) on the walls.

In this work, we choose the temperature of the thermostat, $T_{b}$, as the control parameter, because it is easy to tune it in experiments. The activation energy $E_{A}$ of reaction (1) is used to scale system temperature, $\theta=k_{B} T / E_{A}$. 
We also scale the concentration of species A according to $\alpha=n_{A} /\left(n_{A}+n_{B}\right)$. Then, Eqs. $(3,4)$ can be recast in the following form:

$$
\begin{aligned}
& \frac{d \alpha}{d t}=\sqrt{\theta}\left(-\alpha \exp (-1 / \theta)+K_{2}(1-\alpha)\right) \\
& \frac{d \theta}{d t}=\frac{2}{3} Q \sqrt{\theta}\left(\alpha \exp (-1 / \theta)-K_{0}\left(\theta-\theta_{b}\right)\right)
\end{aligned}
$$

where $t=k_{1}^{0} n_{c} \sqrt{E_{A}} t^{\prime}, K_{2}=p_{2} \kappa^{0} S /\left(k_{1}^{0} n_{C} V\right), Q=q\left(n-n_{C}\right) /\left(E_{A} n\right), K_{0}=$ $\kappa^{0} S /\left(Q k_{1}^{0} n_{C} V\right)$, and $\theta_{b}=k_{B} T_{b} / E_{A}$. Although simple, the model exhibits very rich dynamical behaviors $[27,28,36]$. The bifurcation diagram shown in Fig. 1 presents the domains of stability of the different attractors of the dynamical system described by Eqs. $(8,9)$ as a function of $\theta_{b}$ in the range $[0.19,0.23]$, for the following fixed values of the other parameters: $Q=20$, $K_{0}=0.325$, and $K_{2}=0.3115$. The model has a monotonic nullcline $d \alpha / d t=$ 0 and, an $\mathrm{N}$-shaped nullcline $d \theta / d t=0$ for $\theta_{b}<\frac{1}{4}$. In the phase space $(\theta, \alpha)$, the intersections of the two nullclines define the steady states. In the considered range of $\theta_{b}$, the system possesses either one or three stationary states. 


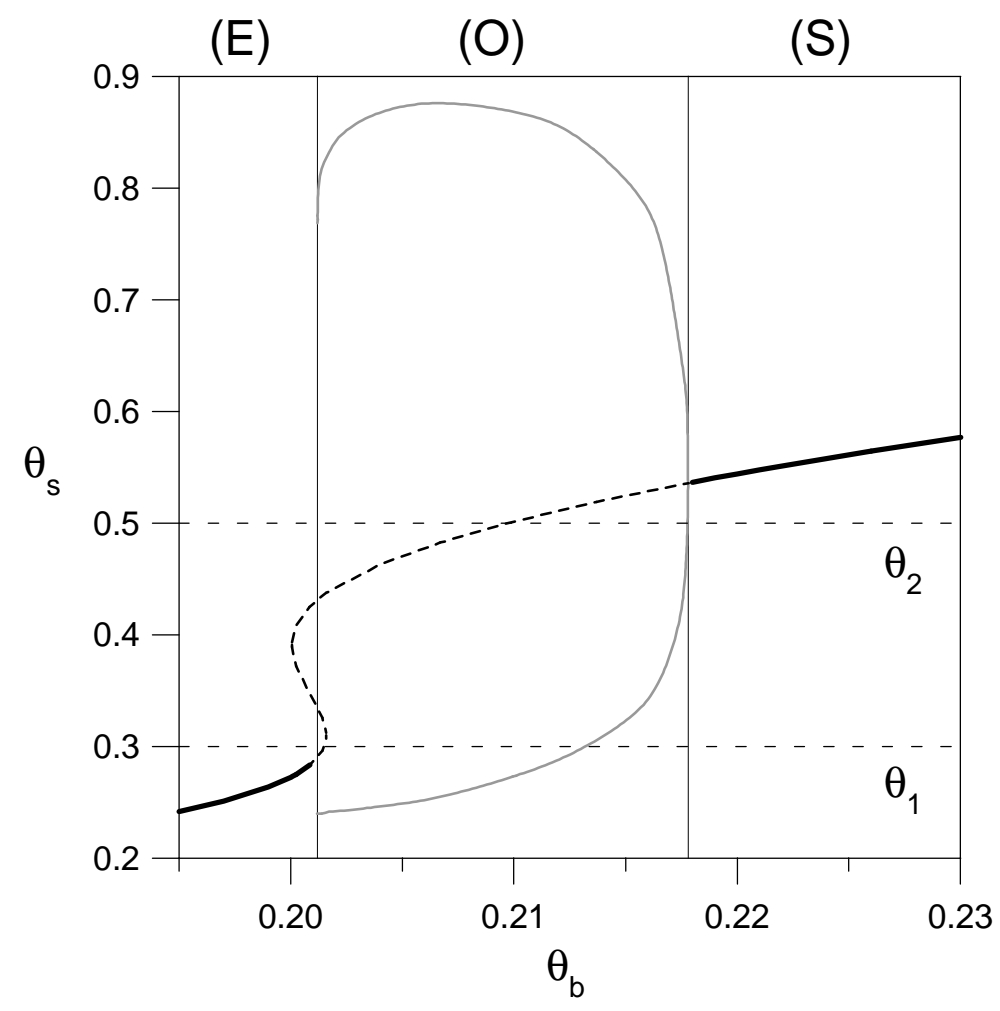

Figure 1: Bifurcation diagram associated with Eqs. $(8,9)$. The two vertical solid lines $\theta_{b}^{\text {homo }}=0.20120583$ and $\theta_{b}^{\text {Hopf }}=0.2178059$ correspond to a homoclinic bifurcation and a Hopf bifurcation, respectively. Domain (E) is associated with excitability, domain $(\mathrm{O})$, with periodic oscillations, and domain (S), with a stable stationary state corresponding to a high temperature. The black solid and dashed lines are the stable and unstable branches of stationary states $\theta_{S}$ versus thermostat temperature $\theta_{b}$, respectively. The gray solid line give the minimum and maximum temperatures reached on the limit cycle. The two thresholds $\theta_{1}=0.3$ and $\theta_{2}=0.5$ introduced in the criterion used to define the interspike interval are represented by horizontal dashed lines. 
As shown in Fig. 1, in the considered range of $\theta_{b}$, there are three domains associated with different types of time evolution. In domain (E), corresponding to low values of $\theta_{b}<0.20120583$, the attractor is a steady state associated with a low temperature value. At the critical value $\theta_{b}^{\text {homo }}=0.20120583$, the system possesses three stationary states including a saddle point and a homoclinic bifurcation occurs: The outgoing separatrix leaving the saddle point meets the incoming separatrix, so that a periodic orbit including the saddle point is formed. In domain $(\mathrm{O})$, defined by $\theta_{b}^{\text {homo }}<\theta_{b}<0.2178059$, the system has a stable limit cycle. The lowest and highest temperatures reached on the cycle at given $\theta_{b}$ are plotted in Fig. 1 . At $\theta_{b}^{\text {Hopf }}=0.2178059$, the system undergoes an inverse Hopf bifurcation and the limit cycle disappears. In domain (S), for $\theta_{b}>\theta_{b}^{H o p f}$, the attractor is a stable focus associated with a high temperature value.

Using the stochastic approach to thermochemical systems that we developed [23], the influence of fluctuations can be analyzed considering a master equation that takes into account both randomness in reactions and heat exchange. The total number of particles $N=N_{A}+N_{B}+N_{C}$ controls the strength of the internal fluctuations. In the stochastic approach, system evolution is described by the probability $P\left(\theta, N_{A}, t\right)$ of finding the system at temperature $\theta$ with $N_{A}$ particles of reactant A at time $t$. The major difficulty with stochastic analysis of a thermochemical model, if compared to a system involving thermoneutral chemical processes associated with discrete particle number jumps, is related to the continuous temperature jumps $\Delta \theta$ associated with Newtonian exchange of energy. The master equation has the 
following integro-differential form [23, 36, 37]

$$
\begin{aligned}
& \frac{\partial}{\partial t} P\left(\theta, N_{A}, t\right)= \\
& \int_{\Delta \theta<\theta} d(\Delta \theta) P\left(\theta-\Delta \theta, N_{A}-\Delta N_{A}, t\right) w\left(\theta-\Delta \theta, N_{A}-\Delta N_{A} \rightarrow \theta, N_{A}\right) \\
& -P\left(\theta, N_{A}, t\right) \int_{\Delta \theta>-\theta} d(\Delta \theta) w\left(\theta, N_{A} \rightarrow \theta+\Delta \theta, N_{A}+\Delta N_{A}\right)
\end{aligned}
$$

in which the transition probability $w$ is composed of three terms:

$$
\begin{aligned}
w\left(\theta, N_{A} \rightarrow \theta+\Delta \theta, N_{A}+\Delta N_{A}\right)= & w_{0}\left(\theta, N_{A} \rightarrow \theta+\Delta \theta, N_{A}\right) \\
& +w_{2}\left(\theta, N_{A} \rightarrow \theta+\Delta \theta, N_{A}+1\right) \\
& +w_{1}\left(\theta, N_{A} \rightarrow \theta+\Delta \theta, N_{A}-1\right)(11
\end{aligned}
$$

The first term, $w_{0}$, is related to the Newtonian exchange of energy without reaction and the two last ones, $w_{2}$ and $w_{1}$, are connected with reactions (2) and (1), respectively. The transition probability $w_{0}$ for exclusive energy exchange is a continuous function of the temperature jump $\Delta \theta$ and does not involve any chemical change. The explicit expression for $w_{0}$ has been derived from the collision rates under the assumption that the velocity distribution of particles remains Maxwellian [23, 37]. Using dimensionless variables, $w_{0}$ can be cast into the following form:

$$
w_{0}\left(\theta, N_{A} \rightarrow \theta+\Delta \theta, N_{A}\right)=\left(\frac{1}{2} N Q K_{0}-N_{B} K_{2}\right) \sqrt{\theta} \omega(\theta, \Delta \theta)
$$

where $\omega(\theta, \Delta \theta)$ is the probability distribution of temperature jumps $\Delta \theta$ at temperature $\theta$ :

$$
\begin{aligned}
\omega(\theta, \Delta \theta)=\frac{\theta \theta_{b}}{\left(\theta+\theta_{b}\right)^{3}}\left(2+\frac{\left(\theta+\theta_{b}\right)\left(\frac{3}{2} N\right)|\Delta \theta|}{\theta \theta_{b}}\right) \\
\quad \times \frac{3}{2} N \begin{cases}\exp \left(-\frac{3}{2} N \frac{|\Delta \theta|}{\theta}\right) & \text { for } \Delta \theta<0 \\
\exp \left(-\frac{3}{2} N \frac{\Delta \theta}{\theta_{b}}\right) & \text { for } \Delta \theta>0\end{cases}
\end{aligned}
$$


Equation (12) does not include the inelastic particle-surface collisions that are related to energy transfer and also to reaction (2). The transition probability $w_{2}$ corresponding to the latter processes describes an increment of $\mathrm{A}$ population by $\Delta N_{A}=1$ combined with a temperature jump, $\Delta \theta$. With the use of Eq. (13), $w_{2}$ can be written as

$$
w_{2}\left(\theta, N_{A} \rightarrow \theta+\Delta \theta, N_{A}+1\right)=N_{B} K_{2} \sqrt{\theta} \omega(\theta, \Delta \theta)
$$

In contrast, the transition probability related to reaction (1) only involves a fixed, temperature increase, $\Delta \theta_{1}=Q /\left(\frac{3}{2} N\right)$, following after the heat release $q$. The decrement of $N_{A}$ associated with reaction (1) is $\Delta N_{A}=-1$. The transition function $w_{1}$ has the standard form [15] following from the frequency of collisions related to reaction (1):

$$
w_{1}\left(\theta, N_{A} \rightarrow \theta+\Delta \theta, N_{A}-1\right)=N_{A} \sqrt{\theta} \exp (-1 / \theta) \delta\left(\Delta \theta-\Delta \theta_{1}\right)
$$

For a fixed number density of A particles the leading terms in the transition probabilities $w_{0}\left(\theta, N_{A} \rightarrow \theta+\Delta \theta, N_{A}\right), w_{1}\left(\theta, N_{A} \rightarrow \theta+\Delta \theta, N_{A}-1\right)$ and $w_{2}\left(\theta, N_{A} \rightarrow \theta+\Delta \theta, N_{A}+1\right)$ are proportional to $N$. In the limit of large particle numbers, the stochastic evolution of the number density of A particles may be performed using Langevin-type equations with a noise term or Langevin force scaling as $N^{-1 / 2}[17]$.

The kinetic Monte Carlo method used to simulate a master equation for discrete jumps is well founded [18] and its appropriate modification for the integro-differential form given in Eq. (10) is presented in detail in reference $[23,28]$. We briefly recall the main steps of the procedure. Initial temperature $\theta$ and initial number of molecules $N_{A}$ are chosen. The escape rate from a 
state $\left(\theta, N_{A}\right)$,

$$
W\left(\theta, N_{A}\right)=\int_{\Delta \theta>-\theta} w\left(\theta, N_{A} \rightarrow \theta+\Delta \theta, N_{A}+\Delta N_{A}\right) d(\Delta \theta)
$$

is computed to select the time $t=-\ln (r) / W$ at which a process takes place, where $r$ is a uniformly distributed random number in the range $[0,1]$. The probabilities of the three possible processes, non-reactive heat exchange, surface reaction (2), and exothermic reaction (1), are considered with relative weights proportional to the corresponding terms in Eq. (11). The system state is then modified according to the selected process and the new transition rates are calculated. The procedure is repeated so that a stochastic time evolution is directly simulated.

\section{Results}

The realization of the stochastic processes given in Eqs. (1,2) from kinetic Monte Carlo simulations of the master equation (Eq. (10)) provides time series for the number $N_{A}$ of A particles and temperature $\theta$. In the following we focus attention on the time evolution of system temperature. 

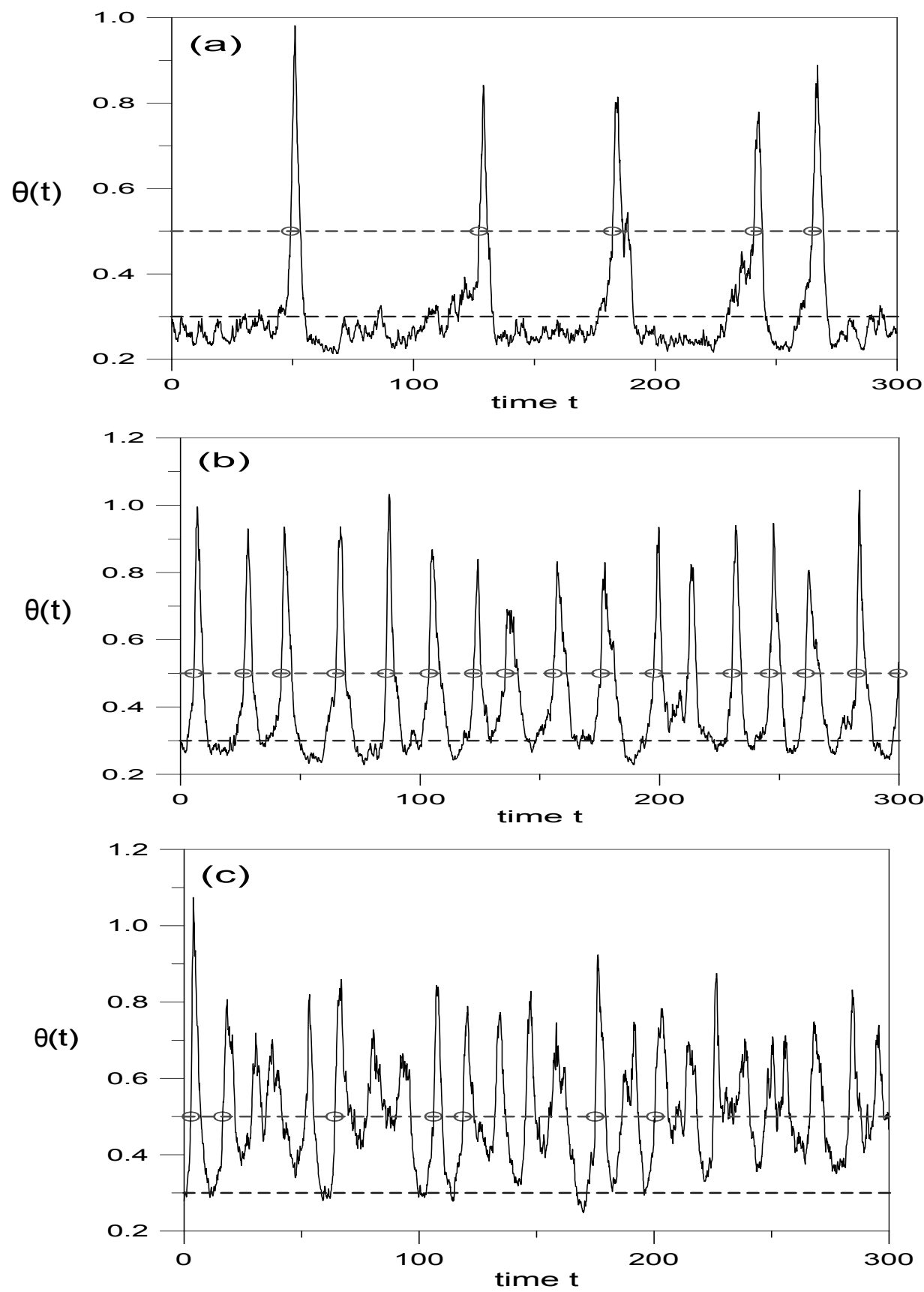

Figure 2: Stochastic time series for temperature deduced from kinetic Monte Carlo simulations of the master equation for a total number of particles $N=5000$ and three thermostat temperatures: (a) $\theta_{b}=0.20$, (b) $\theta_{b}=0.21$, (c) $\theta_{b}=0.22$. The horizontal lines correspond to the threshold values $\theta_{1}$ and $\theta_{2}$ used to define the interspike intervals, whose ends are marked with circles. 
Figure 2a,b,c gives the three typical behaviors obtained in the three domains $(\mathrm{E}),(\mathrm{O})$ and $(\mathrm{S})$ defined in Fig. 1, respectively. In domain (E), where the attractor is a stable steady state, the internal fluctuations activate the random production of spikes and the system is said to be excitable. In domain $(\mathrm{O})$, where the attractor is a limit cycle, temperature presents periodic oscillations perturbed by noise and, in domain $(\mathrm{S})$, where the attractor is a stable focus, temperature fluctuates around the steady state value. In order to characterize the different behaviors, we introduce a single criterion, valid in all three domains and use two temperature thresholds $\theta_{1}<\theta_{2}$. We define the interspike interval $\tau$ as the time between two moments at which temperature $\theta$ becomes larger than $\theta_{2}$ with the condition that $\theta$ drops below $\theta_{1}$ in between. According to Fig. 1, we selected $\theta_{1}=0.3$ and $\theta_{2}=0.5$. Following the results on coherence resonance $[6,9,10]$, we use the mean interspike interval $\langle\tau\rangle$ and its scaled standard deviation $\sigma_{r}$ to characterize the time series of temperature:

$$
\sigma_{r}=\frac{\sqrt{\left\langle\tau^{2}\right\rangle-\langle\tau\rangle^{2}}}{\langle\tau\rangle}
$$

where $\langle\cdot\rangle$ denotes average over the set of interspike intervals deduced from the time series. Alternatively, we can represent the length of an interspike interval using a time unit $\Delta_{t}$ related to the mean value $\langle\tau\rangle$. In the results presented below, we choose $\Delta_{t}=\langle\tau\rangle / 32$. Each interspike interval $\tau$ is mapped onto a number $j$ defined as the integral part of $\tau / \Delta_{t}[30]$. The Shannon entropy $S$ associated with a stochastic trajectory is defined as [31, 38]:

$$
S=-\sum_{j} p_{j} \log _{2}\left(p_{j}\right)
$$

where $p_{j}$ is the probability that an interval of length $j$ is observed. The idea of introducing $S$ in signal processing is to check to which extent complex in- 
formation included in a long time series can be properly captured by a single quantity. 
(a)



(b)

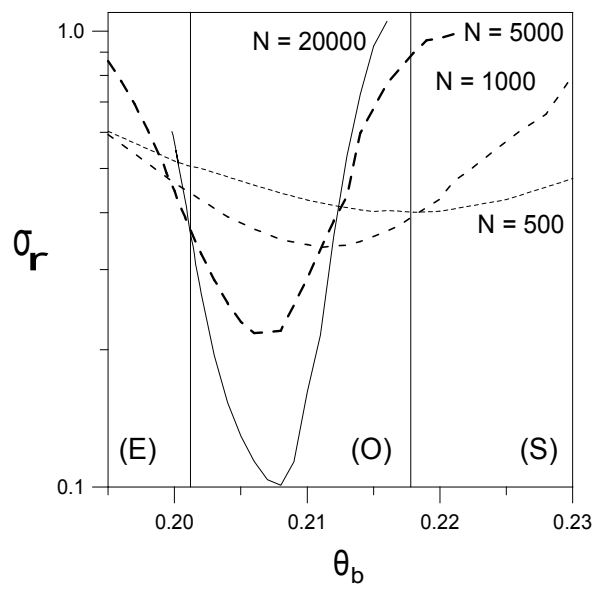

(c)

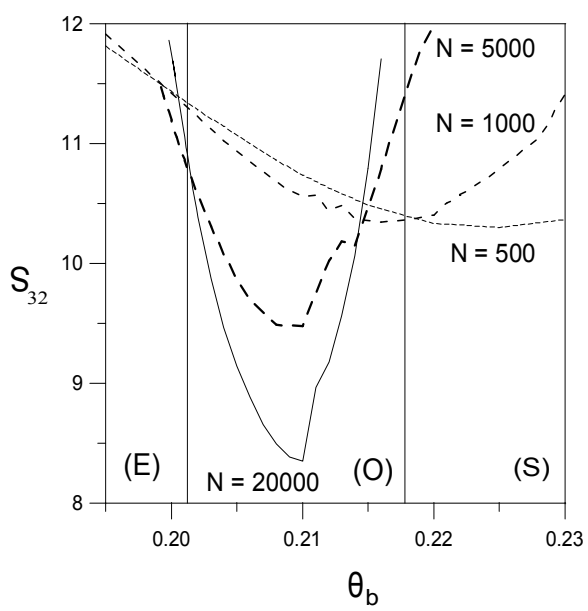

Figure 3: (a) Mean interspike interval $\langle\tau\rangle$, (the thick solid line shows the period of oscillations predicted by the deterministic description using Eqs. $(8,9))$, (b) Standard deviation of the interspike interval scaled by mean value $\sigma_{r}$, and (c) Shannon entropy $S_{32}$ versus thermostat temperature $\theta_{b}$, calculated using $\Delta_{t}=\langle\tau\rangle / 32$, for different total numbers of particles $N$ indicated on the graphs. The two vertical lines indicate the homoclinic and Hopf bifurcations predicted by Eqs. $(8,9)$. 
The statistical characteristics of interspike intervals in the considered thermochemical system as a function of thermostat temperature $\theta_{b}$ is presented in Fig. 3abc. Figure 3a gives the variation of the mean interspike interval $\langle\tau\rangle$ versus thermostat temperature $\theta_{b}$. In the deterministic approach, $\tau$ is only defined in domain $(\mathrm{O})$ as the period of the oscillations associated with the limit cycle. The period $\tau$ deduced from the numerical integration of Eqs. $(8,9)$ in domain $(\mathrm{O})$ diverges at the homoclinic bifurcation for $\theta_{b}^{\text {homo }}$. It monotonically decreases as $\theta_{b}$ increases and vanishes at the Hopf bifurcation for $\theta_{b}^{H o p f}$. In contrast, the mean interspike interval $\langle\tau\rangle$ deduced from the simulation of the master equation can be defined in the three domains. The variation of $\langle\tau\rangle$ versus $\theta_{b}$ deduced from the master equation is nonmonotonic and possesses a minimum. The divergence of $\langle\tau\rangle$ at the homoclinic bifurcation is smoothed with respect to the deterministic approach and a divergence is observed close to the Hopf bifurcation. Actually, as seen in Figs. 2b, it can happen that temperature never drops below the lower threshold $\theta_{1}=0.3$ between two maxima, so that the second maximum is ignored. Hence, in the stochastic approach and in domain $(\mathrm{O})$, close to the disappearance of the limit cycle, the interspike interval is longer than the one predicted by the deterministic equations. In domain $(\mathrm{O})$, the single domain where the deterministic notion of period is meaningful, the agreement between Eqs $(8,9)$ and the master equation becomes better as the number of particles $N$ increases, i.e. as the level of noise decreases. However, due to the criterion chosen to define the interspike interval $\tau$, the agreement is better close to the homoclinic bifurcation than to the Hopf bifurcation. As seen in Fig. 2ac, the lower threshold $\theta_{1}=0.3$ is well adapted to the detection of spikes in the case of 
the excitable system but too demanding in the case of fluctuations around the stable focus for the chosen value of total number of particles. As already mentioned, spikes of too small amplitudes are ignored, which becomes more frequent as fluctuation level decreases, i.e. as total number of particles increases. We could have chosen a criterion leading to a worse agreement close to the homoclinic bifurcation and a better one, close to the Hopf bifurcation. However, the divergence of $\langle\tau\rangle$ close to the Hopf bifurcation is a physical phenomenon which is correctly captured by the chosen definition of the interspike interval. Only the precise value of $\theta_{b}$ for which divergence is observed varies with the adopted thresholds.

The variation of the scaled standard deviation $\sigma_{r}$ of the interspike interval $\tau$ versus thermostat temperature $\theta_{b}$ is given in Fig. $3 \mathrm{~b}$. The curves obtained for different values of the number $N$ of particles possess a minimum which is more pronounced as $N$ increases. As expected, for sufficiently large values of $N \geq 5000$, fluctuation level is low and the most regular, periodic behavior, associated with the minimum of the scaled standard deviation $\sigma_{r}$, is obtained in the middle of the domain of stability of the limit cycle. On the contrary, for a very small number of particles, $N=500$, the minimum is located in domain $(\mathrm{S})$. This behavior is related to noise-induced transitions $[1,6,24,25]$ : In the presence of large internal fluctuations, the domain of stability of the limit cycle is shifted, here, toward higher values of $\theta_{b}$. The comparison between Fig. 3b and Fig. 3c shows that time discretization and Shannon entropy $S$ capture the main features revealed by the scaled standard deviation $\sigma_{r}$. Actually, Shannon entropy vanishes for a delta distribution and 
reaches a maximum for the uniform distribution [38]. Hence, $S$ reflects the width of the distribution of interspike intervals. The good agreement between the variations of $\sigma_{r}$ and $S$ reveals that the time step chosen to compute $S$ is sufficiently small to preserve information. 


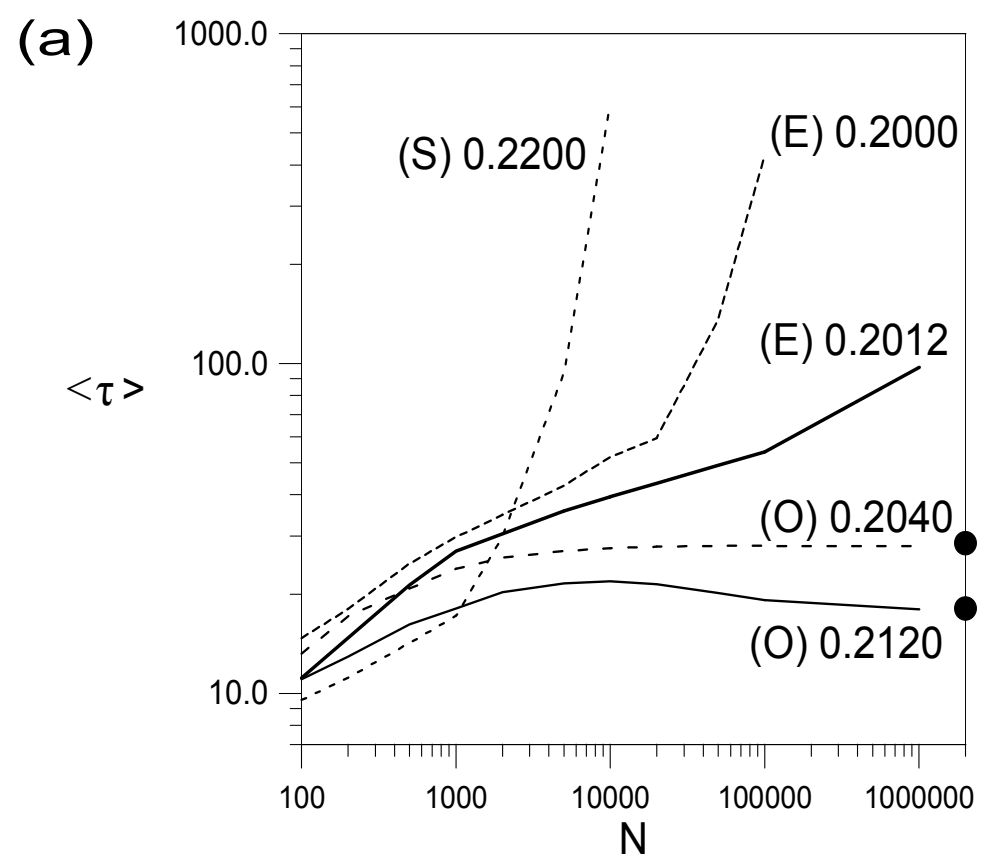

(b)

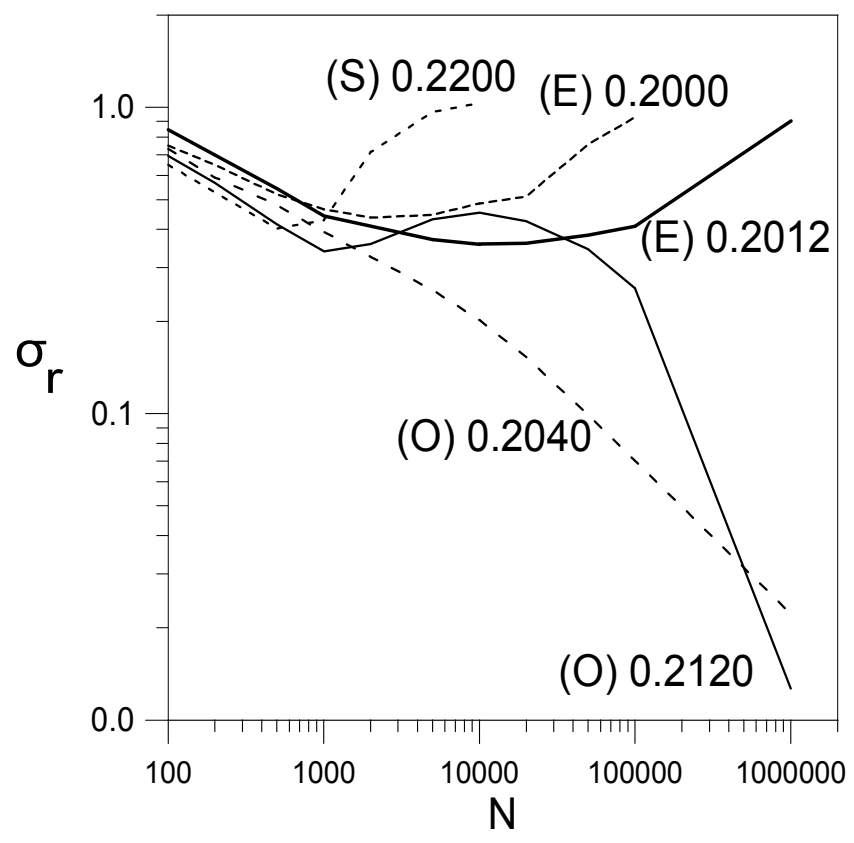

Figure 4: (a) Mean interspike interval $\langle\tau\rangle$, and (b) standard deviation of the interspike interval scaled by mean value, $\sigma_{r}$, as functions of the number of particles $N$ for different thermostat temperatures $\theta_{b}$ indicated on the graphs in the three domains $(\mathrm{E}),(\mathrm{O})$, and $(\mathrm{S})$. 
Figures $4 \mathrm{ab}$ are analogous to Figs. $3 \mathrm{ab}$ but the total number $N$ of particles is considered as the function argument. The mean interspike interval $\langle\tau\rangle$ versus particle number $N$ is shown in Fig 4 a for a few selected value of thermostat temperature $\theta_{b}$. We have selected two values of $\theta_{b}$ in domain (E) including one very close to the homoclinic bifurcation at $\theta_{b}^{\text {homo }}$, two values in domain $(\mathrm{O})$ where the limit cycle is stable, and one value above Hopf bifurcation in domain $(\mathrm{S})$. Outside domain $(\mathrm{O})$, the interspike interval rapidly increases with $N$ as observed in Fig. 3a because the temperature $\theta$ hardly reaches one of the two thresholds $\theta_{1}$ or $\theta_{2}$ in the excitable regime or in the stationary regime as soon as fluctuation level is small. As already mentioned, inside the oscillatory regime, i.e. in domain $(\mathrm{O})$, the values of $\langle\tau\rangle$ converge towards the deterministic period of oscillation for large $N$ values. The behavior of $\langle\tau\rangle$ in domain $(\mathrm{O})$ for $\theta_{b}=0.2120$ presents an interesting maximum about $N=10000$ and the neighborhood of this $\theta_{b}$ value is further investigated in Fig. 5a. As seen in Fig. 1, the specific behavior observed for $\theta_{b}$ close to 0.2130 can be related to the intersection of the lower threshold $\theta_{1}=0.3$ and the decreasing amplitude of the limit cycle at the approach of the Hopf bifurcation. In Fig. 5a, at small $N$, the mean interspike interval is small due to the high fluctuation level enabling the temperature to reach $\theta_{1}$ and $\theta_{2}$ more frequently than once per period. At large $N$, the mean interspike interval converges to the deterministic prediction. For intermediate values of $N$, the fluctuation level is sufficient to modulate the amplitude of the limit cycle so that the threshold $\theta_{1}$ is not crossed at each period and the mean interspike interval possesses a maximum. The range of $\theta_{b}$ values, in which $\langle\tau\rangle$ has a nonmonotonic variation with $N$, depends on the chosen threshold 
$\theta_{1}$, but the existence of a maximum for $\langle\tau\rangle$ versus $N$ for some $\theta_{b}$ value is an intrinsic feature of dynamics. 
(a)

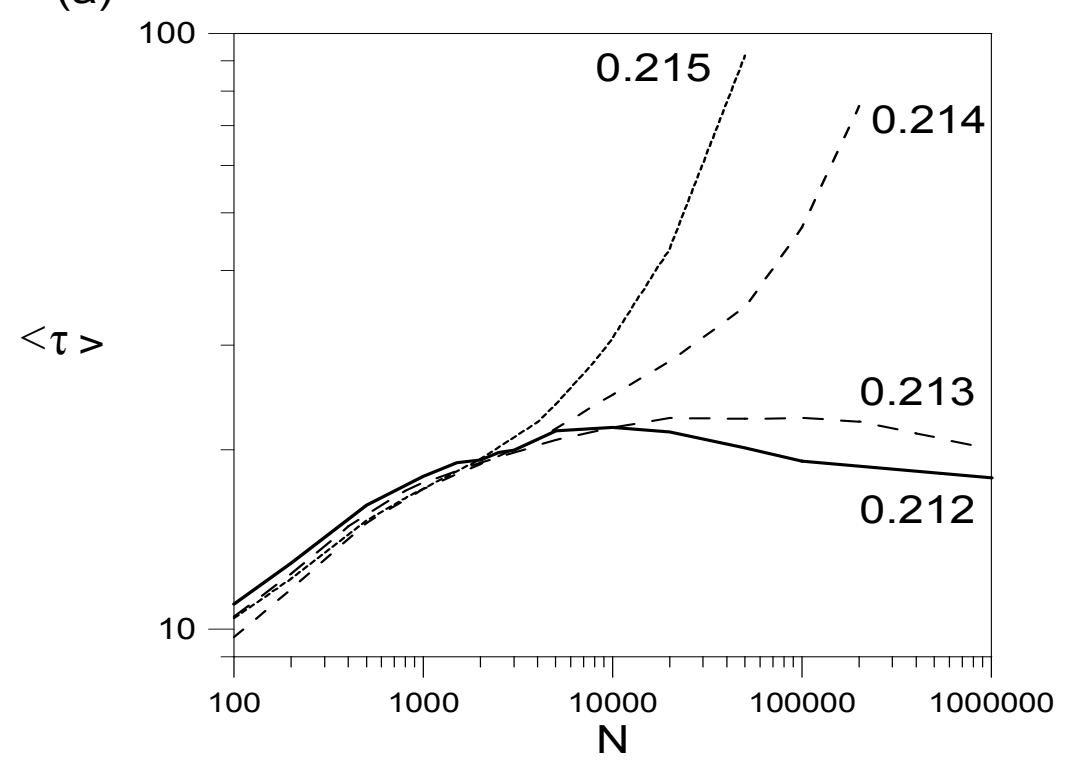

(b)

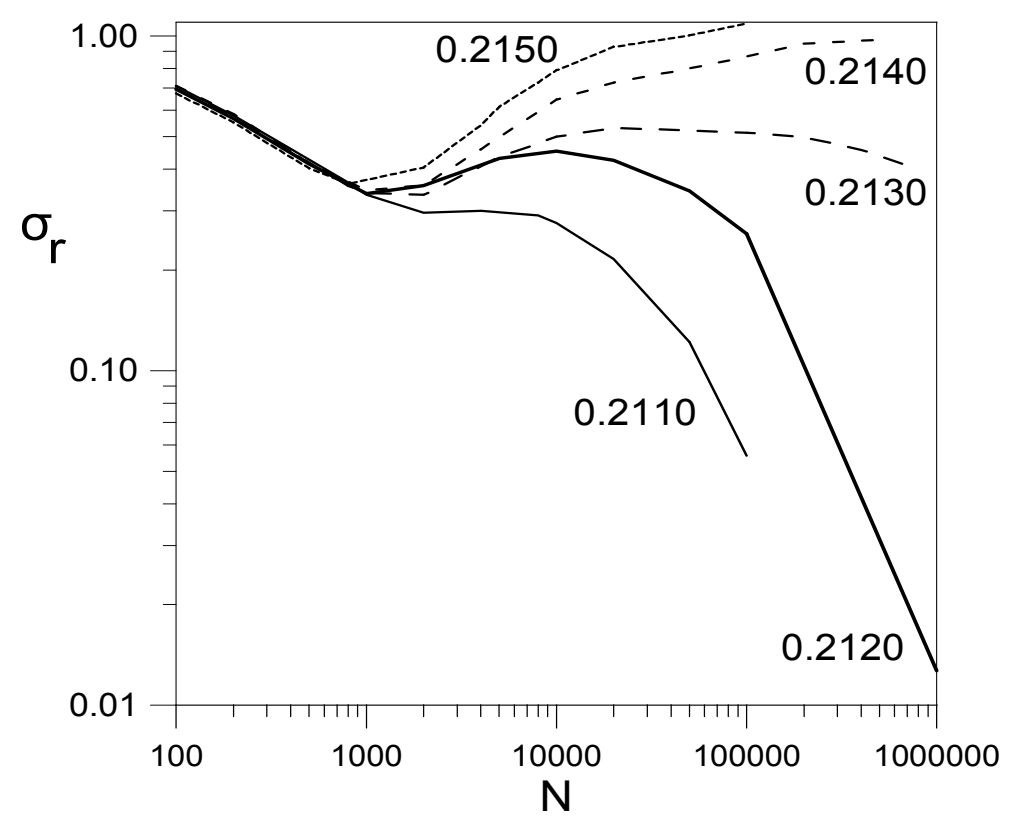

Figure 5: (a) Mean interspike interval $\langle\tau\rangle$, and (b) standard deviation of the interspike interval scaled by mean value, $\sigma_{r}$, as functions of the number of particles $N$ for thermostat different temperature around $\theta_{b}=0.2130$ in domain $(\mathrm{O})$. 
This phenomenon brings an example of stochastic resonance in the oscillatory regime of a thermochemical system. The concept of stochastic resonance has been introduced in the context of climatology as the enhancement of the response of a noisy, bistable system submitted to a periodic forcing $[3,4]$. It has then been extended to certain types of nonlinear systems that are able to optimize their behavior for a well-chosen level of noise. Here the noise level is controlled by the number of particles. In Fig. 5a, the period of oscillations, considered as a function of the number of particles $N$, possesses a maximum in a small range of thermostat temperatures $0.212 \leq \theta_{b} \leq 0.213$. Regarding that the existence of a maximum of a quantity as a function of the noise level is a manifestation of a stochastic resonance $[5,6,7]$, we conclude that the behavior of the period versus $N$ for $\theta_{b}=0.212,0.213$ seen in Fig. 5a shows a new example of stochastic resonance in a highly nonlinear system in which the temperature is one of the dynamical variables.

Fig. $4 \mathrm{~b}$ represents the scaled standard deviation $\sigma_{r}$ of the interspike interval versus particle number $N$ for the same selected values of thermostat temperature $\theta_{b}$ as in Fig. 4a. In domains $(\mathrm{E})$ and $(\mathrm{S}), \sigma_{r}$ possesses a minimum for a given $N$ value, typical of coherence resonance $[8,26]$. For a certain fluctuation level, the appearance of spikes in the excitable regime (domain (E)) is more regular. The interspike interval can be decomposed into two parts, (i) the escape time from the basin of attraction of the stationary state that dominates at large $N$, i.e. for a small fluctuation level, and (ii) the excursion time needed to return to the basin of attraction, which dominates at small $N$. The escape time presents a higher sensitivity to fluctuation 
level than the excursion time. A minimum in the variation of $\sigma_{r}$ versus $N$ is observed for an intermediate fluctuation level, large enough to lead to a negligible activation time but small enough to result in small fluctuations of the excursion time.

In domain $(\mathrm{S})$, an appropriate fluctuation amplitude sustains more regular oscillations around the stable focus, as a reminiscence of the period of the limit cycle: The internal fluctuations unveil a hidden characteristic time scale associated with the imaginary part of the eigenvalues of the system linearized around the focus. The behavior inside domain $(\mathrm{O})$ of stability of the limit cycle is non trivial. In the middle of the domain, for values of $\theta_{b}$ for which the two thresholds $\theta_{1}=0.3$ and $\theta_{2}=0.5$ lie within the amplitude of oscillations, $\sigma_{r}$ monotonically decreases as $N$ increases. The result obtained for $\theta_{b}=0.2040$ is typical of this expected behavior: The appearance of spikes becomes more regular and the fluctuations around the deterministic prediction for the period becomes smaller as $N$ increases. However, for a larger value of thermostat temperature, $\theta_{b}=0.2120$, still inside domain (O) but closer to Hopf bifurcation, the variation of $\sigma_{r}$ versus $N$ possesses a local minimum and a local maximum before a rapid decrease at very large $N$. The asymptotic behavior is consistent with the expected convergence toward negligible fluctuations of $\tau$ around the period of the limit cycle. The minimum of $\sigma_{r}$ for $N \sim 1000$ can be associated with the coherence resonance observed deeper in domain $(\mathrm{O})$ in the oscillatory regime. At larger values of $N$, for $N \sim 10000$, the stochastic resonance identified by the maximum of $\langle\tau\rangle$ for $0.212 \leq \theta_{b} \leq 0.213$ in Fig. 5a induces a maximum of $\sigma_{r}$ in Fig. 5b. As already mentioned, the close values of the lower threshold $\theta_{1}=0.3$ 
and the smallest temperatures reached on the limit cycle lead to random omission of some periods, anomalous increase of some interspike intervals, and consequently, to simultaneous increase of the period and standard deviation. As already mentioned, this phenomenon may appear in a different range of $\theta_{b}$, but will remain a characteristic feature of the system, whatever the choice of $\theta_{1}$. The complex behavior of the scaled standard deviation of interspike interval versus particle number $N$ around thermostat temperature $\theta_{b}=0.2130$ revealed in Fig. $5 \mathrm{~b}$ constitutes a richness of thermochemical systems. With regard to the control of thermochemical systems in nanoreactors, this high variability represents serious risks. The succession of minimum and maximum for close values of $N$ is less marked in the variation of Shannon entropy $S$ given in Figs. 6a for $\Delta_{t}=<\tau>/ 32$. A finer discretization of time scale, $\Delta_{t}=<\tau>/ 64$ instead of $\Delta_{t}=<\tau>/ 32$, slightly increases the entropy of the set of interspike intervals but does not significantly change the dependence of Shannon entropy on $N$. 
(a)

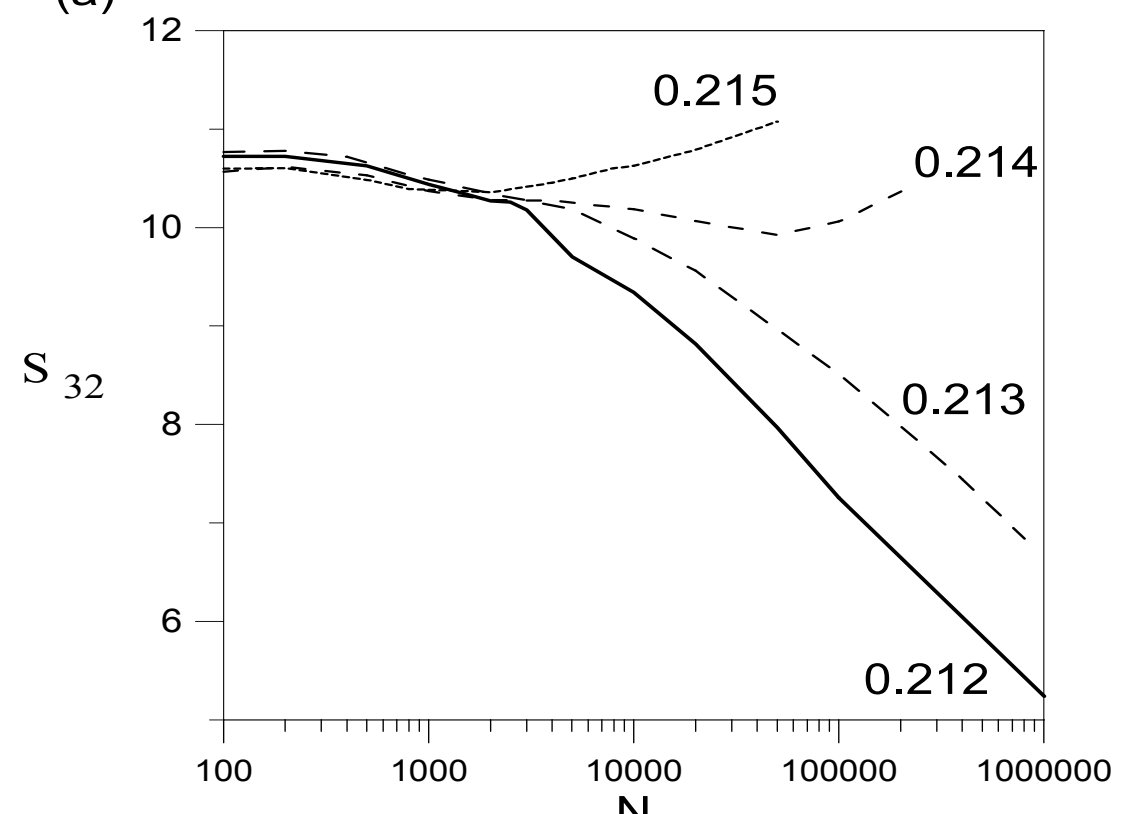

(b)

$\mathrm{N}$

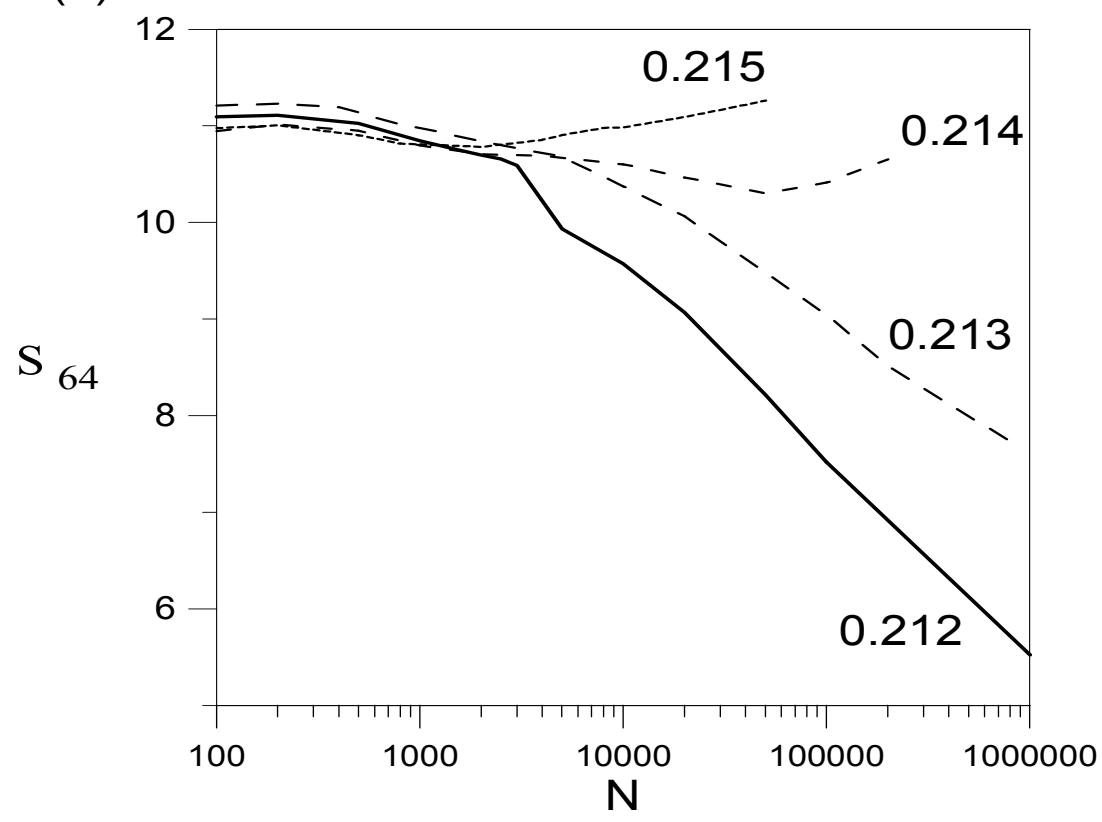

Figure 6: Shannon entropy, (a) $S_{32}$, calculated for $\Delta_{t}=<\tau>/ 32$ and (b) $S_{64}$, calculated for $\Delta_{t}=<\tau>/ 64$ as functions of the total number of particles $N$ for different thermostat temperatures around $\theta_{b}=0.2130$ in domain $(\mathrm{O})$. 


\section{Conclusion}

In this paper, we analyze the stochastic dynamics of a thermochemical system which exhibits a homoclinic bifurcation and a Hopf bifurcation. To this purpose, we use an integro-differential form of the master equation which includes a continuous spectrum of temperature jumps to appropriately deal with energy exchange with a thermostat. The temperature of the thermostat is chosen as the control parameter, easily tunable in experiments. The distribution of interspike intervals deduced from the stochastic time series for temperature is studied in different dynamical regimes including excitability, oscillations and stationarity. At the homoclinic bifurcation, the effect of the internal fluctuations is to smooth the transition toward excitability and to dampen the divergence of the mean interspike interval. At the Hopf bifurcation, a noise-induced transition is correctly predicted by the variation of Shannon entropy $S$ with thermostat temperature for small number of particles $N$, which agrees with the corresponding variation of the scaled standard deviation of interspike interval $\sigma_{r}$ : The minima of $S$ and $\sigma_{r}$, which are associated with the most regular oscillations, are located outside the domain of stability of the limit cycle. The most striking result is obtained for the variation of $\sigma_{r}$ with $N$ in the oscillatory regime close to the Hopf bifurcation: In a small range of thermostat temperatures, the scaled standard deviation of interspike interval possesses a minimum for small $N$ values and a maximum for larger N's. For the chosen coarse graining of time, Shannon entropy is not able to reveal this subtle phenomenon. We interpret the non trivial behavior of $\sigma_{r}$ as the effect of both a coherence resonance and a stochastic resonance. At small $N$, the minimum of $\sigma_{r}$ is related to the coherence resonance also ob- 
served deeper in the domain of stability of the limit cycle in a wider interval of thermostat temperatures: For the optimum noise level, the oscillations are the most regular. For a larger value of the number of particles $N$ (smaller noise amplitude), both the scaled standard deviation of interspike intervals, $\sigma_{r}$, and the mean length of interspike interval, $\langle\tau\rangle$, possess a maximum due to the fact that some of the cycles fail to extend over the assumed threshold levels. The presence of a maximum in the variation of $\langle\tau\rangle$ versus $N$ reveals a stochastic resonance phenomenon regarded as the existence of a maximum of an observable as a function the noise amplitude $[5,6,7]$.

In thermochemical systems and even for very simple chemical schemes, the highly nonlinear dynamics of temperature leads to very rich behaviors that can be controlled by thermostat temperature, making an experimental validation realistic. However, the high variability of the dispersion of interspike intervals, as thermostat temperature or system size slightly varies, suggests that controlling risks in nanoreactors may be a difficult task.

\section{ACKNOWLEDGMENTS}

We acknowledge the support from the French-Polish cooperation program PICS between CNRS and PAN.

\section{References}

[1] W. Horsthemke and R. Lefever, Noise-Induced Transitions, 2nd Ed. (Springer, Berlin, 2006). 
[2] J. S. Hansen, B. Nowakowski, and A. Lemarchand, J. Chem Phys. 124, 034503 (2006).

[3] C. Nicolis, Sol. Phys. 74, 473 (1981).

[4] R. Benzi, A. Sutera and A. Vulpiani, J. Phys. A 14, L453 (1981).

[5] L. Gammaitoni, P. Hänggi, P. Jung, and F. Marchesoni, Rev. Mod. Phys. 70, 223 (1998).

[6] F. Sagues, J. M. Sancho, and J. Garcia-Ojalvo, Rev. Mod. Phys. 79, 829 (2007).

[7] T. Wellens, V. Shatokhin, and A. Buchleitner, Rep. Prog. Phys. 67, 45 (2004).

[8] A. S. Pikovsky and J. Kurths, Phys. Rev. Lett. 78, 775 (1997).

[9] B. Lindner and L. Schimansky-Geier, Phys Rev E 60, 7270 (1999).

[10] B. Lindner, J. Garcia-Ojalvo, A. Neiman, and L. Schimansky-Geier, Phys. Rep. 392, 321 (2004).

[11] J. Escorcia-Garcia, V. Agarwal, and P. Parmananda, Appl. Phys. Lett. 94, 133103 (2009).

[12] P. Hänggi and F. Marchesoni, Rev. Mod. Phys. 81, 387 (2009).

[13] M. Yoshimoto, H. Shirahama, and S. Kurosawa, J. Chem. Phys. 129, 014508 (2008).

[14] A. Lemarchand, B. Nowakowski, G. Dumazer, and C. Antoine, J. Chem. Phys. 134, 034121 (2011). 
[15] G. Nicolis and I. Prigogine, Self-Organization in Nonequilibrium Systems (Wiley, New York, 1977).

[16] N. G. van Kampen, Stochastic Processes in Physics and Chemistry (North-Holland, Amsterdam, 1992).

[17] C. W. Gardiner, Handbook of Stochastic Methods (Springer, Berlin, 2004).

[18] D. T. Gillespie, Annu. Rev. Phys. Chem. 58, 35 (2007).

[19] G. Nicolis, F. Baras, and M. Malek Mansour, in Nonlinear Phenomena in Chemical Dynamics, edited by A. Pacault (Springer, Berlin, 1981), p. 104.

[20] G. Nicolis and M. Malek Mansour, Phys. Rev. A 29, 2845 (1984).

[21] F. Baras, Ph.D. thesis, Université Libre de Bruxelles, 1985.

[22] R. D. Present, Kinetic Theory of Gases (McGraw-Hill, New York, 1958).

[23] B. Nowakowski and A. Lemarchand, Phys. Rev. E 64, 061108 (2001).

[24] A. Lemarchand and B. Nowakowski, Physica A 331, 409 (2004).

[25] A. Lemarchand and B. Nowakowski, Mol. Simulat. 30, 773 (2004).

[26] O. V. Ushakov, H. J. Wunsche, F. Henneberger F, I. A. Khovanov, L. Schimansky-Geier, and M. A. Zaks, Phys. Rev. Lett. 95, 123903 (2005).

[27] A. Lemarchand and B. Nowakowski, J. Phys.: Condens. Matter 19, 065130 (2007). 
[28] A. Kolbus, A. Lemarchand, A. Kawczynski, and B. Nowakowski, Phys. Chem. Chem. Phys. 12, 13224 (2010).

[29] B. Nowakowski, A. Kawczynski, A. Kolbus and A. Lemarchand, Eur. Phys. J. B 84, 137 (2011).

[30] J. Gorecki, A. Gorecki, A. Lemarchand, and B. Nowakowski, Eur. Phys. J. B 86, 264 (2013).

[31] T. M. Cover and J. A. Thomas, Elements of Information Theory (WileyInterscience, 2006)

[32] K. Miyakawa and H. Isikawa, Phys Rev E 66, 046204 (2002).

[33] V. Beato, I. Sendina-Nadal, I. Gerdes I, and H. Engel, Philos. Transact. A Math. Phys. Eng. Sci. 366, 381 (2008).

[34] A. L. Kawczynski and J. Gorecki, J. Phys. Chem. 96, 1060 (1992).

[35] B. V. Volter and I. Y. Salnikov, Modeling and Optimization of Catalytic Processes (Nauka, Moscow, 1965) (in Russian).

[36] A. L. Kawczynski and B. Nowakowski, Phys. Chem. Chem. Phys. 10, 289 (2008).

[37] B. Nowakowski and A. Lemarchand, Physica A 311, 80 (2002).

[38] I. Goychuk and P. Hänggi, Phys. Rev. E 61, 4272 (2000). 